(1)

CrossMark

\title{
Will we be singing a different tune on combined post- and pre-capillary pulmonary hypertension?
}

\author{
Steven $\mathrm{Hsu}^{1}$ and Ryan J. Tedford ${ }^{2}$ \\ Affiliations: ${ }^{1}$ Johns Hopkins University, Cardiology, Baltimore, MD, USA. ${ }^{2}$ Medical University of South \\ Carolina, College of Medicine, Charleston, SC, USA. \\ Correspondence: Ryan J. Tedford, Medical University of South Carolina, Strom Thurmond Gazes Building, \\ Room 215, 114 Doughty St/MSC592, Charleston, SC 29425, USA. E-mail: TedfordRamusc.edu
}

@ERSpublications

More uncertainty in treating CpcPH with PAH specific therapy http://ow.ly/W2XU30hMwGl

Cite this article as: Hsu S, Tedford RJ. Will we be singing a different tune on combined post- and precapillary pulmonary hypertension? Eur Respir J 2018; 51: 1702589 [https://doi.org/10.1183/ 13993003.02589-2017].

There have been many well-intended pursuits in medicine that should have panned out, but did not. Case in point: premature ventricular complexes (PVC) post-myocardial infarction were noted to be associated with increased risk of death, and thus the Cardiac Arrhythmia Suppression Trial (CAST) Study investigated if pharmacological PVC suppression with class I anti-arrhythmics would reduce the rate of arrhythmic death [1]. Surprisingly, the study showed that although PVCs were successfully suppressed, there were excess deaths due to arrhythmia and shock in such patients treated with encainide or flecainide [1].

Perhaps along similar lines, there is on-going controversy regarding the treatment of group II pulmonary hypertension $(\mathrm{PH})$, or $\mathrm{PH}$ due to left heart disease (PH-LHD). Group II PH is defined by a mean pulmonary artery pressure (mPAP) of $\geqslant 25 \mathrm{mmHg}$ with a pulmonary artery wedge pressure (PAWP) of $>15 \mathrm{mmHg}$. However, a subset of these patients also have a pre-capillary component, defined by a diastolic pulmonary gradient $(\mathrm{DPG}) \geqslant 7 \mathrm{mmHg}$ and/or pulmonary vascular resistance (PVR) $>3$ Wood units (WU) [2]. Combined post- and pre-capillary $\mathrm{PH}(\mathrm{CpcPH})$ is not only common in group II PH [2], but numerous studies have shown it confers increased risk of mortality in the LHD population beyond isolated post-capillary $\mathrm{PH}(\mathrm{IpcPH})$ [3, 4]. Enticingly, multiple pulmonary vasodilator therapies exist for the treatment of pre-capillary $\mathrm{PH}$ associated with group I pulmonary arterial hypertension (PAH). Whether such therapies should be used to treat the pre-capillary component of PH-LHD remains the topic of much debate [5].

There exists a strong pathobiologic rationale for therapeutically targeting $\mathrm{CpcPH}$ with $\mathrm{PAH}$-specific drugs. In the setting of passive increases in pulmonary pressure in PH-LHD, endothelial dysfunction leads to reduced nitric oxide, enhanced endothelin-1, pulmonary arterial vasoconstriction [6], and even pulmonary arterial remodelling $[7,8]$. Recently, AssAD et al. $[9,10]$ found patients with $\mathrm{CpcPH}$ had a genetic profile that more closely resembled that of PAH than of IpcPH. In support of treatment, some relatively small studies using prostanoids, endothelin receptor antagonists (ERAs), and phosphodiesterase-5 (PDE5) inhibitors have shown acute improvement in symptoms, haemodynamics, and exercise capacity [5]. The PDE5 inhibitor sildenafil has the most supportive data, with several single-centre, randomised controlled

Received: Dec 122017 | Accepted after revision: Dec 202017

Conflict of interest: R.J. Tedford has received personal fees (for the Hemodynamic Core Lab for SOPRANO study) from Actelion, outside the submitted work.

Copyright CERS 2018 
trials showing an improvement in exercise haemodynamics and functional capacity in LHD populations with and without PH [11-13], alongside other studies demonstrating reductions in PVR in patients awaiting heart transplant or after mechanical circulatory support $[14,15]$.

Data also exist to the contrary. The aforementioned sildenafil trials should be interpreted with caution, as all were single-centre trials. The multicentred PhosphodiesteRasE-5 Inhibition to Improve CLinical Status and EXericse Capacity in Diastolic Heart Failure (RELAX) trial did not find improvement in peak oxygen consumption after 12 weeks of sildenafil treatment in patients with heart failure with preserved ejection fraction (HFpEF) [16], many of whom had PH. Other PAH drugs including epoprostenol and bosentan have been studied in LHD with disappointing or adverse results $[17,18]$, with bosentan notable for causing increased oedema [18].

However, although results are mixed for the use of PAH-specific therapies in PH-LHD, it would be hard to discount their use in $\mathrm{CpcPH}$ altogether. It has been difficult to interpret $\mathrm{PAH}$-specific treatment studies in LHD and PH-LHD since many have studied heterogeneous populations or lacked a haemodynamic diagnosis of PH-LHD upon enrolment [5]. If the totality of these studies to date have taught us anything, it is that $\mathrm{CpcPH}$ and $\mathrm{IpcPH}$ are very different entities, as are heart failure with preserved and reduced ejection fraction (HFrEF), and that the successful study of these entities requires invasive haemodynamic confirmation.

The Macitentan in subjects with combined prE- and post-capiLlary pulmonary hypertension due to left ventricular DYsfunction (MELODY-1) trial, a phase II study reported in this issue of the European Respiratory Journal, is thus immensely important in the field [19]. It is the first study to look at the use of an ERA specifically in patients with $\mathrm{CpcPH}$, and one of the few PH-LHD trials to require invasive diagnostic confirmation. With these characteristics alone, MELODY-1 defines its study cohort better than the vast majority of existing PH trials in PH-LHD. In this study, Vachiery and colleagues enrolled 63 patients across multiple sites and continents into a randomised, double-blind, placebo-controlled trial of macitentan versus placebo for the treatment of $\mathrm{CpcPH}$ in LHD. Patients were formally defined with $\mathrm{CpcPH}$ using right heart catheterisation to document $\mathrm{mPAP} \geqslant 25 \mathrm{mmHg}$, PAWP $>15 \mathrm{mmHg}$, and PVR $\geqslant 3 \mathrm{WU}$ and DPG $\geqslant 7 \mathrm{mmHg}$. Although it was a phase II study primarily focused on safety end-points (namely, fluid retention or change in functional capacity), the authors also looked at exploratory secondary end-points of efficacy, most notably change in PVR. Overall, they find a non-statistically significant increase in fluid retention in the macitentan arm, as well as a higher number of patients who discontinued study treatment in the macitentan arm. Perhaps most notably, they did not find a significant different in PVR after 12 weeks of treatment, with a similar decrease noted in both arms.

Several important strengths of this study merit highlighting. First, the authors required an invasive haemodynamic diagnosis of $\mathrm{CpcPH}$ as well as post-treatment haemodynamic measures, which in itself is a significant undertaking. How to define $\mathrm{CpcPH}$ haemodynamically remains debated [20], yet impressively, the carefully designed inclusion and exclusion criteria led to enrolment of a cohort that clearly had significant CpcPH, with an average PVR of 5.8 WU, TPG of $27 \mathrm{mmHg}$, and DPG of $10 \mathrm{mmHg}$. As such, the conclusions we can glean from MELODY-1 surpass those that can be made from similar prior studies. In this context, it is important to note, and perhaps disappointingly so, that PVR did not decrease in the macitentan arm, and actually decreased similarly in both arms. Although a phase II study would be underpowered to detect differences, it is striking that macitentan failed to show even a trend of lowering PVR. Perhaps its mechanism of action is not optimal for lowering PVR in a CpcPH cohort, yet we also recall that in the RELAX study, sildenafil also failed to lower total right ventricular afterload [21]. The reason for PVR decrease in the control arm is also unclear, but vascular decongestion from heart failure therapy alone can reduce PVR [22], and perhaps trial enrolment led to more rigorous heart failure assessment and treatment in the placebo group. Any PVR conclusions are made with the phase II caveat, but the rigorous definition of $\mathrm{CpcPH}$ and the near absence of signal would argue this is still worth noting.

The other important item to note is the increased fluid retention and functional decline in the macitentan arm. Although macitentan theoretically has less propensity for fluid retention than bosentan [23], the fact that a signal was noted in a small study are relevant in light of the ENABLE study, which noted, in a much larger cohort, significantly greater fluid retention in the first 2 to 4 weeks of bosentan [18]. In MELODY-1 study there was a $10 \%$ greater rate of fluid retention or worsening functional capacity in the macitentan arm, coupled with a significantly greater rate of treatment discontinuation for any reason. Such data will be important to bear in mind with future ERA studies in PH-LHD. These results also remind us that the use of PAH-specific therapies in PH-LHD should only be in the context of clinical trials, as duly noted by the authors.

The odds were perhaps stacked against finding benefit for macitentan, as it was not powered for such. Macitentan was also studied here in a primarily older HFpEF cohort, with concomitant high proportions 
of obesity, hypertension, and atrial fibrillation, which are comorbidities that can worsen right ventricular-pulmonary vascular coupling and make the search for treatment benefit difficult. Macitentan may still prove more useful in a larger cohort of $\mathrm{CpcPH}$, or in specific $\mathrm{CpcPH}$ or LHD sub-populations. For instance, patients with scleroderma and LHD may also suffer from concomitant group I PAH pathology, and it remains to be seen if they could benefit from PAH specific therapy [24].

Thus, the present study raises some important caution to the notion of using ERAs for the treatment of $\mathrm{CpcPH}$ in PH-LHD. The lack of therapeutic benefit (no PVR decline) coupled with potential harm (increase in fluid retention) sounds similar to the failed experiments of anti-arrhythmics in post-myocardial infarction arrhythmia. That said, while the current results may be disheartening, not all hope is lost for the use of PAH-specific therapies in PH-LHD. More work remains to be done in the study of macitentan as well as other PAH-specific therapies in PH-LHD. The most important aspect of MELODY-1 is that by rigorously defining $\mathrm{CpcPH}$ using right heart catheterisation, the authors set a new standard in the design and interpretation of pulmonary vasodilator studies in PH-LHD. This standard will hopefully inform the design of future studies to come.

\section{References}

1 Echt DS, Liebson PR, Mitchell LB, et al. Mortality and morbidity in patients receiving encainide, flecainide, or placebo. The Cardiac Arrhythmia Suppression Trial. N Engl J Med 1991; 324: 781-788.

2 Galiè N, Humbert M, Vachiery J-L, et al. 2015 ESC/ERS Guidelines for the diagnosis and treatment of pulmonary hypertension: The Joint Task Force for the Diagnosis and Treatment of Pulmonary Hypertension of the European Society of Cardiology (ESC) and the European Respiratory Society (ERS). Eur Heart J 2015: ehv317.

3 Miller WL, Grill DE, Borlaug BA. Clinical features, hemodynamics, and outcomes of pulmonary hypertension due to chronic heart failure with reduced ejection fraction: pulmonary hypertension and heart failure. JACC Heart Fail 2013; 1: 290-299.

4 Tampakakis E, Leary PJ, Selby VN, et al. The diastolic pulmonary gradient does not predict survival in patients with pulmonary hypertension due to left heart disease. JACC Heart Fail 2015; 3: 9-16.

5 Vachiery J-L, Adir Y, Barbera JA, et al. Pulmonary hypertension due to left heart diseases. J Am Coll Cardiol 2013; 62: 25 Suppl, D100-D108.

6 Cheli M, Vachiery J-L. Controversies in pulmonary hypertension due to left heart disease. F1000Prime Rep 2015; 7: 07.

7 Delgado JF, Conde E, Sánchez V, et al. Pulmonary vascular remodeling in pulmonary hypertension due to chronic heart failure. Eur J Heart Fail 2005; 7: 1011-1016.

8 Gerges C, Gerges M, Lang MB, et al. Diastolic pulmonary vascular pressure gradient: a predictor of prognosis in "out-of-proportion" pulmonary hypertension. Chest 2013; 143: 758-766.

9 Assad TR, Brittain EL, Wells QS, et al. Hemodynamic evidence of vascular remodeling in combined post- and precapillary pulmonary hypertension. Pulm Circ 2016; 6: 313-321.

10 Assad TR, Hemnes AR, Larkin EK, et al. Clinical and biological insights into combined post- and pre-capillary pulmonary hypertension. J Am Coll Cardiol 2016; 68: 2525-2536.

11 Lewis GD, Shah R, Shahzad K, et al. Sildenafil improves exercise capacity and quality of life in patients with systolic heart failure and secondary pulmonary hypertension. Circulation 2007; 116: 1555-1562.

12 Guazzi M, Tumminello G, Di Marco F, et al. The effects of phosphodiesterase-5 inhibition with sildenafil on pulmonary hemodynamics and diffusion capacity, exercise ventilatory efficiency, and oxygen uptake kinetics in chronic heart failure. J Am Coll Cardiol 2004; 44: 2339-2348.

13 Guazzi M, Samaja M, Arena R, et al. Long-term use of sildenafil in the therapeutic management of heart failure. J Am Coll Cardiol 2007; 50: 2136-2144.

14 Jabbour A, Keogh A, Hayward C, et al. Chronic sildenafil lowers transpulmonary gradient and improves cardiac output allowing successful heart transplantation. Eur J Heart Fail 2007; 9: 674-677.

15 Tedford RJ, Hemnes AR, Russell SD, et al. PDE5A inhibitor treatment of persistent pulmonary hypertension after mechanical circulatory support. Circ Heart Fail 2008; 1: 213-219.

16 Redfield MM, Chen HH, Borlaug BA, et al. Effect of phosphodiesterase-5 inhibition on exercise capacity and clinical status in heart failure with preserved ejection fraction: a randomized clinical trial. JAMA 2013; 309: 1268-1277.

17 Califf RM, Adams KF, McKenna WJ, et al. A randomized controlled trial of epoprostenol therapy for severe congestive heart failure: The Flolan International Randomized Survival Trial (FIRST). Am Heart J 1997; 134: 44-54.

18 Packer M, McMurray JJV, Krum H, et al. Long-term effect of endothelin receptor antagonism with bosentan on the morbidity and mortality of patients with severe chronic heart failure: primary results of the ENABLE trials. JACC Heart Fail 2017; 5: 317-326.

19 Vachiéry J-L, Delcroix M, Al-Hiti H, et al. Macitentan in pulmonary hypertension due to left ventricular dysfunction. Eur Respir J 2018; 51: 1701886.

20 Guazzi M, Naeije R. Pulmonary hypertension in heart failure: pathophysiology, pathobiology, and emerging clinical perspectives. J Am Coll Cardiol 2017; 69: 1718-1734.

21 Hussain I, Mohammed SF, Forfia PR, et al. Impaired right ventricular-pulmonary arterial coupling and effect of sildenafil in heart failure with preserved ejection fraction: an ancillary analysis from the Phosphodiesterase-5 Inhibition to Improve Clinical Status And Exercise Capacity in Diastolic Heart Failure (RELAX) Trial. Circ Heart Fail 2016; 9: e002729.

22 Kalogeropoulos AP, Vega JD, Smith AL, et al. Pulmonary hypertension and right ventricular function in advanced heart failure. Congest Heart Fail 2011; 17: 189-198.

23 Pulido T, Adzerikho I, Channick RN, et al. Macitentan and morbidity and mortality in pulmonary arterial hypertension. N Engl J Med 2013; 369: 809-818.

24 Hsu S, Houston BA, Tampakakis E, et al. Right ventricular functional reserve in pulmonary arterial hypertension. Circulation 2016; 133: 2413-2422. 